\section{Artifacts And Non-Local Effects In SPM Potential Measurements}

\author{
Sergei V. Kalinin and Dawn A. Bonnell \\ University of Pennsylvania \\ sergei2@seas.upenn.edu
}

In the last few years Scanning Probe Microscopy (SPM) has become one of the primary tools of science and technology. In addition to topographical imaging, surface potential, conductivity, optical, ferroelectric and magnetic properties that can be studied down to the nanometer level [1]. However, quantitative and sometimes qualitative studies of fundamental physical phenomena in meso-and nanoscale systems are often hindered by SPM imaging artifacts. Here we briefly discuss the principles of operation and the major sources of artifacts in electrostatic measurements by SPM.

One of the most well known techniques for local potential imaging is Scanning Surface Potential Microscopy (SSPM). SSPM is based on dual pass imaging. The grounded tip acquires surface topography during the first pass. During the second pass, the tip retraces the topographic profile maintaining a constant tip-surface separation of 10 to $1000 \mathrm{~nm}$ above the surface. In SSPM the tip is biased directly by $V_{\mathrm{tp}}=V_{\mathrm{do}}+V_{\mathrm{ac}} \cos (\omega t)$, where $V_{\mathrm{ac}}$ is referred to as the driving voltage. The first harmonic of the capacitive force acting on the tip is

$$
F_{I \omega}(z)=F_{l o c}\left(V_{d c}-V_{s}\right) V_{a c},
$$

where $F_{100}$ is tip-surface capacitance gradient, $z$ is tip-surface separation and $V_{s}$ is surface potential. Feedback is used to nullify the first harmonic of the force by adjusting the $\mathrm{DC}$ component of tip bias, and mapping the nulling potential $V_{o c}$ yields a surface potential map. Here, the SPM tip acts as a moving Kelvin probe, hence the alternative name for this technique as Kelvin Probe (Force) Microscopy.

The artifacts in the surface potential measurements can be traced either to the non-idealities of microscope electronics and feedback effects or to the probe effects. The latter include the effects of tip geometry and non-local cantilever effects and effects of surface topography. Here we briefly discuss the relative significance of these effects.

One of the possibilities for erroneous potential measurements in SSPM are DC and AC voltage drops in the measurement device. DC voltage drops in the electronics can be important if the tip-surface resistance is very small. One of the weakest links in the measurement set-up is that the contact between the tip holder and tip substrate typically has resistances on the of order of several $\mathrm{kOhm}$ and larger, depending on the type of tip coating. However, in SSPM tip-surface separation is large, therefore resistance is also large ( $>\mathrm{GOhm}$ ) and DC voltage drops in the electronics are negligible (unless there are problems with insulation, etc). If the tip accidentally touches the surface so that there is a significant current flow the effects are easy to notice: for a nonconductive or contaminated metal surface a charged patch will form. Overall, DC leakage is negligible with a possible exception for extremely small tip-surface separations, in which case tip-surface charge transfer is possible. This is not quite true for $\mathrm{AC}$ leakage. Modest capacitive coupling between the AC biased channel and the rest of the microscope on the order of $\sim \mathrm{nF}$ at the typical frequencies of 10 to $100 \mathrm{kHz}$ is equivalent to a leakage resistance on the order of 10 to $100 \mathrm{kOhm}$. For cantilevers with semiconductive coatings and large probe-tip holder contact resistances this leakage resistance can be (but usually is not) smaller than the contact resistance between tip holder and the substrate, resulting in significant attenuation of driving voltage. This effect can be compensated by improving probe holder contact, e.g. by placing a drop of silver paint or a piece of Indium on the contact area [2].

Other sources of error in potential measurements are feedback effects and non-local cantilever effects. Due to feedback non-ideality, the first harmonic of the cantilever response is not nullified, rather it is reduced to some small, but finite value. Also, simple calculations suggest that at typical tip surface separations on the order of 10 to $100 \mathrm{~nm}$, tip-surface and cantilever-surface capacitive gradients are comparable [3]. Therefore, both contributions are important and the signal picked up by the probe can be subdivided into the local tip part and non-local cantilever part. In this case, the nulling condition in SSPM corresponds to

$$
F_{l o c}\left(V_{d c}-V_{s}\right)+F_{n l}\left(V_{d c}-V_{a v}\right)=\delta / V_{a c}
$$

where $F_{\text {loc }}$ is the local part of the tip surface capacitance gradient, $F_{n i}$ is the non-local and cantilever part, $V_{\mathrm{s}}$ is the local potential below the tip, $V_{\mathrm{av}}$ is the surface potential averaged over the cantilever length, and $\delta$ is the feedback constant (which, of course, depends on the gain values for feedback loop). The nulling voltage $V_{\text {dc }}$ is then

$$
V_{d c}=V_{c} \frac{F_{l u c}}{F_{l o c}+F_{n t}}+V_{a v} \frac{F_{u t}}{F_{l o c}+F_{n l}}+\frac{\delta}{V_{u c}\left(F_{l o x}+F_{n t}\right)} \text { Eq. } 3
$$

Reliable measurement of local surface potential is possible if and only if $F_{\mid 00} \gg F_{\mathrm{nl}^{\prime}}$, i.e., the tip is close to the surface, and the third term is small, i.e., the feedback error is minimized. Noteworthy is that the relative potential variations across the surface are independent of feedback effects, i.e., the measured potential difference between two points relates to the true potential difference as:

$$
\left(V_{1}-V_{2}\right)_{\text {measived }}=\left(V_{1}-V_{2}\right) \frac{F_{l o c}}{F_{l o c}+F_{n l}} \quad \text { Eq. } 4
$$

The absolute surface potential value, however, depends on feedback effects.

$$
V_{d c}=V_{a t}+\frac{\delta}{V_{u c}\left(F_{t o c}+F_{n l}\right)}
$$

The implications of Eqs. 4 and 5 are two fold. Measurements of absolute surface potential (or contact potential difference) are subject to errors due to the feedback effect. The reciprocal dependence of absolute surface potential on driving voltage can be easily confirmed experimentally by variation of driving amplitude [3]. Doing so at different tip-surface separations allows the effect of $F_{10}$ and $F_{n t}$ to be determined and true surface potential can be obtained from the analysis of the data [4]. At the same time, potential variations across the surface do not depend on $V_{\mathrm{ac}}$ (except that small driving amplitudes result in the increased noise level), but the effective potential difference between two spots on the surface (say, 10 to 40 micrometers apart) decays logarithmically with lift height. For very large tip-surface separations, the potential contrast along the surface disappears. $[2,5]$

Of course, more complex electronic artifacts are possible. For example, capacitive crosstalk between a tip bias channel and photodiode detector channel will result in an error signal proportional to the driving voltage and this effect can be minimized only by decreasing the tip surface separation, but not by increasing driving amplitude. 

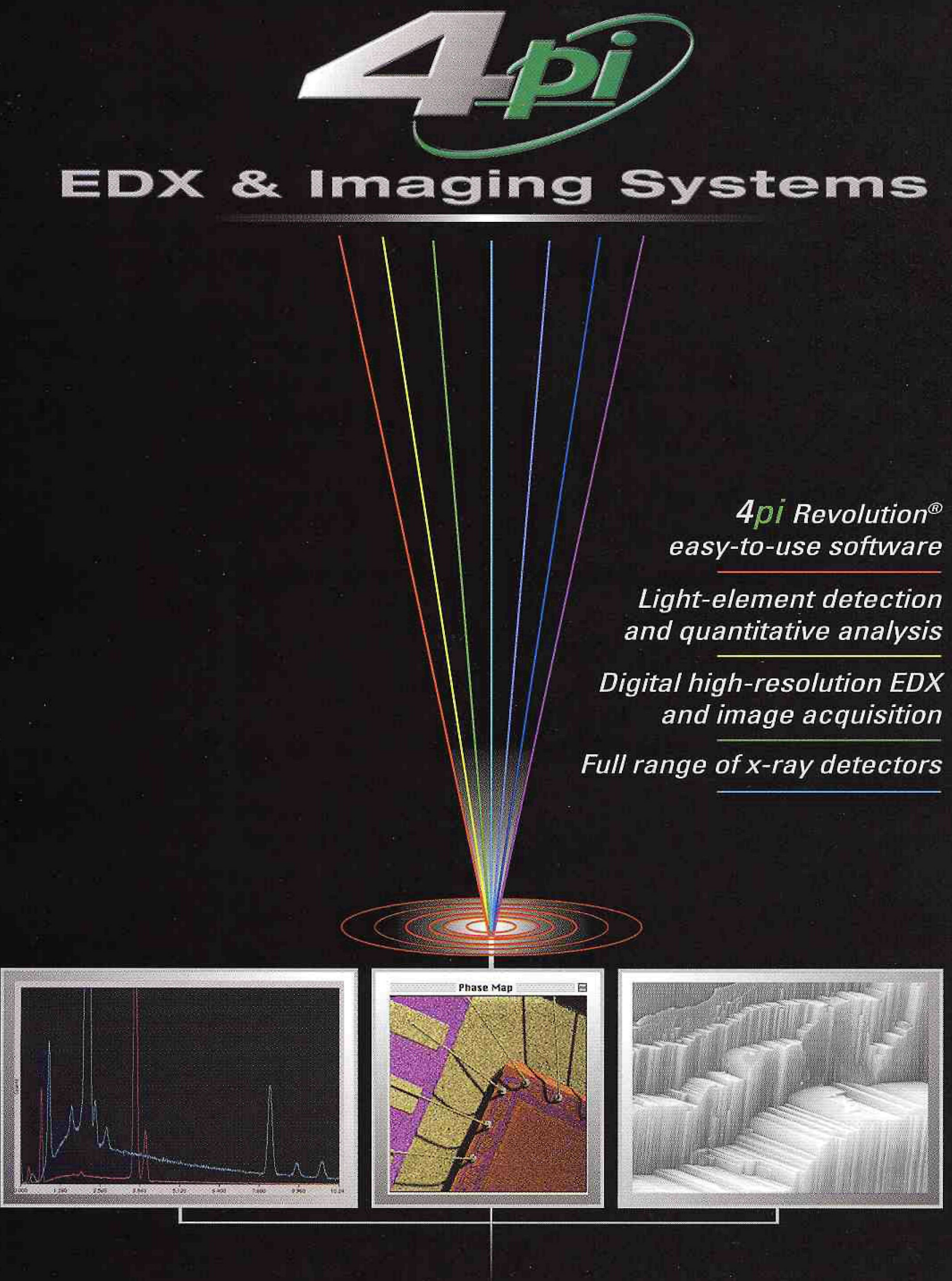
Other sources of artifacts in surface potential measurements are geometric inhomogeneities of the surface and tip-induced surface phenomena. The cross talk between potential and topographic images is well known [6] and a number of algorithms to reduce this effect have been undertaken [7]. A very interesting, and almost unstudied issue is the tip effect on surface properties. [8] Voltage or mechanical modulation of the biased tip induces $A C$ current in the region directly below the tip proportional to $V d C / d t+C d V / d t$, where the first term originates from oscillation in tip position and second from voltage oscillations. For a voltage modulated tip with a driving amplitude of 10 $\mathrm{V}$, driving frequency of $100 \mathrm{kHz}$ and a (heavily underestimated) tip surface capacitance of $10 \mathrm{aF}$, the corresponding displacement current amplitude is $\sim 100 \mathrm{pA}$. Assuming the lateral size of the biased region to be $\sim 100 \mathrm{~nm}$, variations in surface potential due to the displacement current become significant for resistivities higher than $10^{-2} \mathrm{Ohm} \mathrm{m}$. For well conducting surfaces or semiconductor surfaces with pinned Fermi levels, changes in surface potential due to displacement current are negligible. However, this might not be the case for surfaces with unpinned Fermi levels, where tip-induced band bending and associated variations in surface potential can be important. Another interesting case is imaging of nanoscale objects, for which injection of even several electrons can severely affect properties. This analysis has a number of interesting implications for lateral transport measurements on biased nanoscale devices.

Similar analysis can be performed for other electrostatic SPMs such as Electrostatic Force Microscopy [1]. For EFM measurements in the frequency detection mode, the signal is proportional to the second derivative of the tip surface capacitance rather than the first, as in SSPM/KPFM. Therefore, the signal is more local and simple numerical estimates suggest that the cantilever effect can be ignored. However, the capacitive force gradient between the tip and the surface measured in EFM renders this technique extremely sensitive to the topographic structure of the surface. To measure local potential by EFM a number of images must be collected for different tip biases. The local potential can be determined from the apex of parabolic dependence between frequency shift and tip bias rendering it relatively inconvenient for quantitative studies. Of course, there are also a number of feedback artifacts in EFM, however, the difficulties in the quantitative interpretation of EFM data makes the detailed analysis of these artifacts less relevant.

\section{Endnotes:}

[1] S.V. Kalinin and D.A. Bonnell, in Scanning Probe Microscopy and Spectroscopy: Theory, Techniques and Applications, ed. D.A. Bonnell (Wiley VCH, New York, 2000, p. 205), and references therein.

[2] Digital Instruments listserver, spm@ di.com

[3] Sergei V. Kalinin and Dawn A. Bonnell, Phys. Rev. B 63, 125411 (2001).

[4] In ambience, mobile surface charges and adsorbtion all contribute to measured potential. However, here we consider only measurement artifacts.

[5] G. Koley, M. G. Spencer, and H. R. Bhangale, Appl. Phys. Lett. 79, 545 (2001).

[6] It can be easily demonstrated by acquiring topographic and potential images on the clean rough gold surface: topographic protrusions are associated with depressions on potential images.

[7] A. Efimov and S.R. Cohen, J. Vac. Sci. Technol. A 18, 1051 (2000).

[8] This is a well known problem for magnetic force microscopy: tip magnetization can distort surface magnetization in the magnetically soft samples. For potential imaging, the relevant reference is: $Y$. Leng, C. C. Williams, L. C. Su, and G. B. Stringfellow, Appl. Phys. Lett. 66, 1264 (1995).

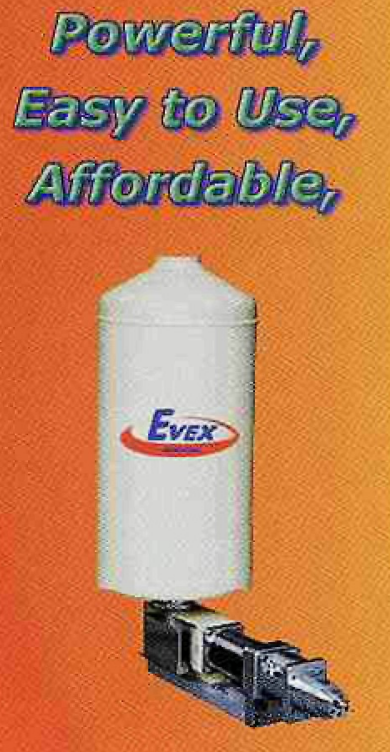

Poweritul

Instralls on any Electron Microscope

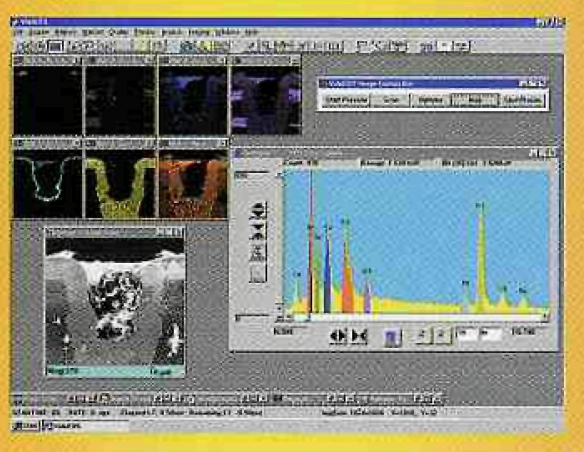

Detectors Kroay Mileroanalysis (Digital Thuaging Elemencal Mapping
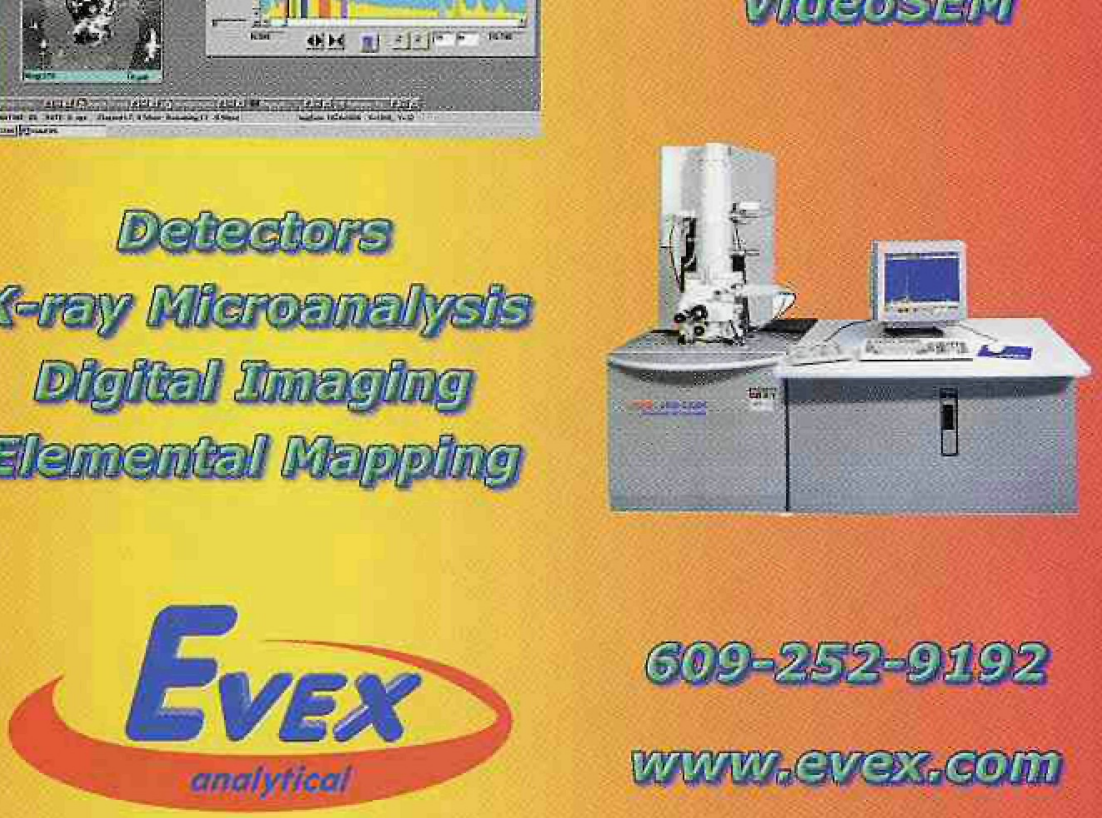
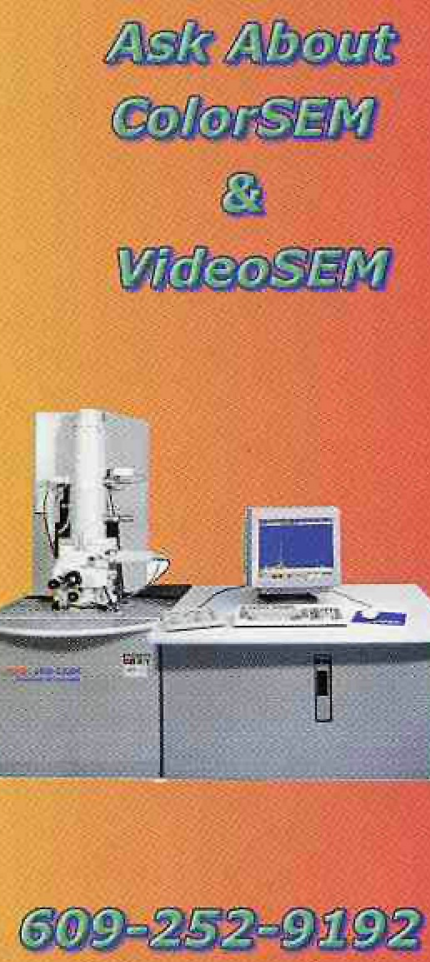

WWWW ever3.som 


\section{SPI Supplies.}

The complete source for all your microscopy needs...

\section{just a click away. www.2spi.com}

Visit SPI Supplies to view the complete on-line catalog with up-to-the-minute product and pricing information.

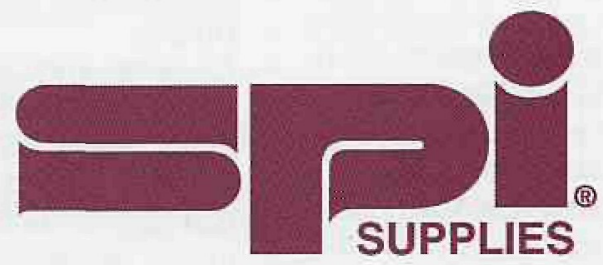

SPI Supplies Division of STRUCTURE PROBE, Inc.

P.0. Box 656 - West Chester, PA 19381-0656 USA

Phone: 1-610-436-5400 • 1-800-2424-SPI (USA and Canada) • Fax: 1-610-436-5755 • E-mail: sales@2spi.com 
Monday, August 5th

PLATFORM SESSIONS (AM)

8:00-11:45 Applications and Developments of Focused Ion Beams

8:00-12:00 Electron Cryo-microscopy of Biological Macromolecules

8:00-10:15 Polymer Characterization: It's Not Just For Microscopes Anymore

8:00-12:00 Practical Applications of Metallography 8:30-12:15 Aberration Correction in TEM and STEM and its Application to Real-World Materials-A Symposium Honoring the Contributions of Albert Crewe and Harald Rose

8:30-12:00 Advances and Applications in Vascular Corrosion Casting in Microvascular Research

8:30-12:00 Advances in Nanoscale Technology

8:30-12:00 Tutorial: Basic Confocal Microscopy

8:30-11:45 Electron Crystallography and Quantitative Electron Diffraction

8:30-12:00 Quantitative X-Ray Microanalysis in the Microprobe and in the SEM: Theory and Practice

8:30-12:00 Scanning Probe Microscopy: Technical Advances and Applications

9:00-12:00 In Situ Electron Microscopy Techniques and Applications/ Reactions

POSTER PRESENTATIONS, 1:00-3:00PM

- Aberration Correction in TEM and STEM and its Application to Real-World Materials-A Symposium Honoring the Contributions of Albert Crewe and Harald Rose

- Advances in Nanoscale Technology

- Applications and Developments of Focused lon Beams

- Biomedical Applications

- Current Topics in Low Voltage SEM

- Electron Cryo-microscopy of Biological Macromolecules

- Electron Crystallography and Quantitative Electron Diffraction Hirsch Symposium: In Situ Electron Microscopy Techniques and Applications/ Reactions

- Microbiology

- Phase Transformation in Metals, Alloys and Ceramics

- Practical Applications of Metallography

- Quantitative X-Ray Microanalysis in the Microprobe and in the SEM: Theory and Practice

- Scanning Probe Microscopy: Technical Advances and Applications

PLATFORM SESSIONS (PM)

3:00-5:15 Aberration Correction in TEM and STEM and its Application to Real-World Materials-A Symposium Honoring the Contributions of Albert Crewe and Harald Rose

3:00-4:30 Advances and Applications in Vascular Corrosion Casting in Microvascular Research

3:00-4:45 Advances in Nanoscale Technology

3:00-5:00 Applications and Developments of Focused Ion Beams

3:00-4:45 Electron Cryo-microscopy of Biological Macromolecules

3:00-4:45 Electron Crystallography and Quantitative Electron Diffraction
3:00-4:00 Hirsch Symposium: In Situ Electron Microscopy

Techniques and Applications/Reactions

3:00-4:45 Practical Applications of Metallography

3:00-4:45 Quantitative X-Ray Microanalysis in the Microprobe and in the SEM: Theory and Practice

3:00-4:00 Tutorial: Practical Methods for Transmission Electron Microscopy of Polymers

4:00-5:00 Tutorial: Scanned Probe Microscopy

3:00-4:00 Technologists' Forum Special Topics: Immunology 101: Back to Basics

\section{PRESIDENTIAL HAPPENINGS}

5:00 Harnessing The Power Of Adobe Photoshop 7

Tuesday, August 6th

\section{PLATFORM SESSIONS (AM)}

8:00-12:00 Confocal and Deconvolution for Biologists

8:15-12:00 Advances in Nanoscale Technology

8:15-12:00 Plant-microbes Interactions at the Cellular and Molecular Levels

8:30-12:00 3-D Electron Microscopy of Macromolecules

8:30-12:00 Electron Crystallography and Quantitative Electron Diffraction

8:45-12:00 Electron Holography, Interference Phenomena and Related Techniques: A Symposium Honoring the Contributions of Hannes Lichte and Akira Tonomura

8:45-12:00 Elmar Zeitler Symposium: Analytical Electron Microscopy-Past and Future

8:30-12:00 Microscopy, Microanalysis and Image Analysis in the Pharmaceutical Industry

8:30-11:30 Microscopy and Microanalysis of Self-Organized Soft Condensed Matter

8:30-12:00 Quantitative X-Ray Microanalysis in the Microprobe and in the SEM: Theory and Practice

8:30-11:00 Problem Solving with the Experts: Addressing Issues in Digital Imaging for the Microscopist: II

9:00-11:00 How to be a MICRO Volunteer-A Practical Workshop About Educational Outreach

9:00-12:00 Hirsch Symposium: In Situ Electron Microscopy Techniques and Applications/Reactions

9:00-12:00 Microstructural Examination and Imagery of Engineering Materials

11:00-12:00 Microscopy for Canadian Schools-A discussion of Ways and Means

POSTER PRESENTATIONS, 1:00-3:00PM

- 3-D Electron Microscopy

- Botany

- Confocal and Deconvolution for Biologists

- Correlative Microscopy

- Elmar Zeitler Symposium: Analytical Electron Microscopy-Past and Future

- Geology/Mineralogy

- Image Simulation and Image Processing Techniques

- Microstructural Examination and Imagery of Engineering Materials

- Electron Holography, Interference Phenomena and Related Techniques: A Symposium Honoring the Contributions of Hannes Lichte and Akira Tonomura 
- Plant-microbes Interactions at the Cellular and Molecular Levels

- Semiconductors

- Teaching and Learning, Creating Effective, Innovative Solutions in Microscopy, Imaging and Analysis

\section{PLATFORM SESSIONS (PM)}

3:00-4:15 3-D Electron Microscopy of Macromolecules

3:00-5:00 Confocal and Deconvolution for Biologists

3:00-4:00 Tutorial: Electron Backscatter Diffraction in the

SEM: Orientation Mapping and Phase Identification for Materials Science

3:00-5:00 Electron Holography, Interference Phenomena and Related Techniques: A Symposium Honoring the Contributions of Hannes Lichte and Akira Tonomura

3:00-4:30 Hirsch Symposium: In Situ Electron Microscopy

Techniques and Applications/Reactions

3:00-5:00 Interfaces

3:00-5:00 Microstructural Examination and Imagery of

Engineering Materials

3:00-5:00 Microscopy, Microanalysis and Image Analysis in the Pharmaceutical Industry

4:00-5:00 Tutorial: Quality Systems for Microscopy and Microanalysis: ISO 9000 and More

\section{PRESIDENTIAL HAPPENINGS}

5:00 The 1st 10 Million Years of Solar System History: Application of Microbeam Techniques to the Study of Meteorites

\section{Wednesday, August 7th}

\section{PLATFORM SESSIONS (AM)}

8:00-12:00 Current Topics in Low Voltage SEM

8:00-12:00 EELS and EFTEM Analysis

8:00-12:00 Electron Backscatter Diffraction of Materials:

Geology to Nanotechnology

8:00-12:00 Electron Holography, Interference Phenomena and Related Techniques: A Symposium Honoring the Contributions of Hannes Lichte and Akira Tonomura

8:00-11:45 Industrial Applications of Microscopy-Techniques for the Real World

8:00-12:00 Magnetic Materials and Super-conducting Materials

8:00-12:00 Metallography and Microstructural Evaluation of Contemporary Materials

8:00-11:45 Modulated Structures and Quasicrystals

8:00-12:00 State of the Art Infrared and Raman Microanalysis

8:30-12:00 Advances in Microwave Technology - Creating a Revolution in Biological Specimen Processing for Light and Electron Microscopy

8:30-11:45 Biominerals

8:30-11:45 Special Staining Techniques for Biological/ Materials Samples

8:45-12:00 Advances in Ultrastructural and Non-invasive Imaging of Skin

9:00-10:00 Tutorial: Spectral Imaging: Getting the Most from All that Data

\section{POSTER PRESENTATIONS, 1:00-3:00PM}

- Advances in Linking Structure to Function in Biomaterials

- Biological Ultrastructure (Cells, Tissues, Organ Systems)

- Biominerals

- Corporate Members Session

- EELS and EFTEM Analysis
- Electron Backscatter Diffraction of Materials: Geology to Nanotechnology

- Image Contrast Mechanisms in the Variable Pressure SEM: the New Imaging Dimension

- Industrial Applications of Microscopy-Techniques for the Real World

- Magnetic Materials and Super-Conducting Materials

- Metallography and Microstructural Evaluation of Contemporary Materials

- Modulated Structures and Quasicrystals

- New Developments in Immunolabelling

- Pathology

PLATFORM SESSIONS (PM)

3:00-5:00 Advances in Microwave Technology-Creating a Revolution in Biological Specimen Processing for Light and Electron Microscopy

3:00-5:00 Advances in Ultrastructural and Non-invasive Imaging of Skin

3:00-5:00 Current Topics in Low Voltage SEM

3:00-5:00 Magnetic Materials and Super-conducting Materials

3:00-5:00 Metallography and Microstructural Evaluation of Contemporary Materials

3:00-4:45 Pathology

3:00-5:00 Problem Solving with the Experts: Core Facility Management

3:00-5:00 Special Staining Techniques for Biological/ Materials Samples

Thursday, August 8th

\section{PLATFORM SESSIONS (AM)}

8:00-12:00 The Microstructural Approach to Food Processing and Engineering

8:00-12:00 EELS and EFTEM Analysis

8:00-12:00 Frontiers of X-ray Spectrometry

8:00-12:00 Teaching and Learning, Creating Effective, Innovative Solutions in Microscopy, Imaging and Analysis

8:00-12:00 Industrial Applications of Microscopy-Techniques for the Real World

8:00-12:00 Magnetic Materials and Super-conducting Materials

8:00-9:45 State of the Art Infrared and Raman Microanalysis 8:30-11:45 Image Contrast Mechanisms in the Variable

Pressure SEM: the New Imaging Dimension

8:30-12:15 New Developments in Immunolabelling

9:00-12:00 Advances in Linking Structure to Function in Biomaterials

9:00-12:00 Electron Microscopy of Macro-, Micro- and MesoPorous Materials

8:30-9:30 Tutorial: Cryo-EM of Large Complexes

9:30-10:30 Tutorial: Cellular Dynamics using AFM

11:00-12:00 Tutorial: Electron Tomography of Biological

Structures

10:30-12:00 Technologists' Forum Roundtable Discussion:

Legal and Ethical Issues of Data Ownership

PLATFORM SESSIONS (PM)

3:00-5:00 EELS and EFTEM Analysis

3:00-5:00 Electron Microscopy of Macro-,Micro- and Meso-

Porous Materials

3:00-5:00 Magnetic Materials and Super-conducting Materials

3:30-5:00 State of the Art Infrared and Raman Microanalysis 